\title{
Mäzenatentum in Deutschland. Eine Chance für die Stadtentwicklung?
}

\author{
B. Faller and C.-C. Wiegandt \\ Geographisches Institut der Universität Bonn, Bonn, Germany \\ Correspondence to: C.-C. Wiegandt (wiegandt@uni-bonn.de)
}

\begin{abstract}
Received: 18 December 2014 - Revised: 2 September 2015 - Accepted: 21 September 2015 - Published: 28 October 2015
Kurzfassung. The article deals with the growing importance of philanthropy regarding German urban development in recent years. Between the opposing poles of financial shortage of public authorities and increasing concentration of private assets, the financial and local engagement of wealthy citizens open up creative leeway for urban policy. Two nationwide surveys in 467 cities respectively 5000 foundations in 22 cities as well as 24 qualitative interviews with various decision makers from policy, administration, economy and foundation system provide the empirical basis for this article. Key findings of the article are two systematisations. One systematisation categorizes German cities regarding their level of philanthropy activity. The other systematisation outlines different project types concerning the strategy and procedure of cooperation between philanthropists and local authorities.
\end{abstract}

\section{Ausgangssituation und Zielsetzung}

Der finanzielle Handlungsrahmen deutscher Städte und Gemeinden hat sich in den letzten Jahren verschlechtert. Politik und Verwaltung suchen deshalb nach neuen Formen für die Finanzierung stadtentwicklungspolitischer Aufgaben auf allen räumlichen Maßstabsebenen. So erscheint es willkommen, wenn Projekte, die im öffentlichen Interesse liegen, durch ein finanzielles Engagement aus der Bürgerschaft flankiert werden. Mit den immer begrenzter werdenden finanziellen Möglichkeiten der Kommunen hat gleichzeitig die Einkommens- und Vermögensungleichheit in Deutschland in den vergangenen Jahren zugenommen.

Im Jahr 2008 gibt es rund 800.000 Millionäre in Deutschland (Rickens, 2011). Für die Städte und Gemeinden können wohlhabende Mäzene eine besondere Rolle bei der Bewältigung gesellschaftlicher Aufgaben spielen. Mäzene stellen in einzelnen Städten zum Teil große Summen ihres Privatkapitals zur Verfügung, ohne dafür eine unmittelbare Gegenleistung zu erhalten. Anerkennung und Ansehen, Dankbarkeit und Bewunderung sind in solchen Fällen schwer messbare Gegenwerte, die als „Tauschobjekte“ für Investitionen in die gebaute Umwelt oder auch in die Schaffung sozialer Infrastrukturen fungieren.
Schenkungen dieser Art erfolgen in der gesamten Breite des kommunalen Aufgabenspektrums. So werden soziale oder kulturelle Einrichtungen von Mäzenen unterstützt, Veranstaltungen oder Feste subventioniert, oder die gebaute Umwelt einer Stadt oder Gemeinde gezielt finanziert. Ebenso vielseitig wie die thematische Bandbreite an Vorhaben sind auch die Organisationsformen, in denen sich solche Schenkungen vollziehen. Die Spannweite reicht von Einzelpersonen bis zu verschiedenen Formen der Stiftungen sowie speziellen Fundraising-Organisationen, die die Mäzene für ein größeres Einzelvorhaben zusammenbringen.

In welchen Städten spielt ein solches Mäzenatentum eine Rolle in Deutschland? Wie ist das finanzielle Engagement der Mäzene in diesen Städten zu erklären? Welche Bedeutung und welchen Umfang hat das lokale mäzenatische Handeln? Warum engagieren sich Mäzene für ihre Stadt? In welchem Zusammenhang stehen die mäzenatischen Projekte zu den städtischen Aufgaben und ihren Akteuren? Wo gibt es Interessenkonflikte?

Ziel des Beitrages ist es, mit der Beantwortung dieser Fragen den unterschiedlichen Stellenwert des Mäzenatentums in deutschen Städten herauszuarbeiten und strategische Interaktionen zwischen den involvierten Akteuren zu analysieren. Zum einen sind es die unterschiedlichen Bedeutungen des Mäzenatentums in deutschen Städten, die unser For- 
schungsinteresse leiten. Zum anderen liegt unser Interesse in den fallspezifischen Akteurskonstellationen, in denen es um Machtverhältnisse und politische Interaktionen geht, die in den konkreten Projekten beim Umgang mit Mäzenen zum Tragen kommen. Eine eigene Theorie zum Mäzenatentum streben wir mit unserem Beitrag nicht an. Im Vordergrund stehen vielmehr die gesellschaftspolitische Einordnung des Mäzenatentums und eigene empirische Befunde, mit denen wir die Ausprägungen und Folgen einer zunehmend als neoliberal bezeichneten Stadtpolitik aus der Perspektive der Governance-Forschung aufzeigen wollen. Unsere Ergebnisse beruhen auf einem gemeinsamen DFG-Projekt, das wir zwischen 2010 und 2013 bearbeitet und das wir bereits mit dem Fokus auf unsere Fallstudien zu den Konflikten beim Zusammenspiel von Stadtpolitik, kommunaler Verwaltung und Bürgerschaft an anderer Stelle dargestellt haben (Faller, 2011; Faller und Wiegandt, 2010, 2015). In diesem Beitrag stehen deshalb in Abgrenzung zu den bereits publizierten Erkenntnissen zum einen Typisierungen der Städte hinsichtlich der Rolle von Mäzenen und zum anderen Typisierungen der Projektkonstellationen im Vordergrund des Interesses.

\section{Mäzene als neue Akteure der Stadtentwicklung in Zeiten des Neoliberalismus}

Seit den 1990er Jahren werden unter dem Schlagwort des Neoliberalismus schleichende Veränderungen in der Gesellschaft in Deutschland diskutiert. Kritisiert wird aus dieser Perspektive, dass eine wohlfahrtsstaaliche Politik zugunsten der marktwirtschaftlichen Selbststeuerung eingeschränkt wird (Heinz, 2015:10ff). Das als neoliberal bezeichnete Gesellschaftsmodell (Berndt, 2014) ist im Gegensatz zum vorhergehenden fordistischen Modell durch eine zunehmende Privatisierung, Liberalisierung und staatliche Deregulierung charakterisiert. Die wachsende soziale Ungleichheit (BMAS, 2013), die sich in zunehmenden Disparitäten auf allen räumlichen Maßstabsebenen niederschlägt (Wiegandt, 2015:4ff), wird ebenfalls als Folge dieser Neuausrichtung interpretiert (Heinz, 2015:25f). Im vierten Armuts- und Reichtumsbericht der Bundesregierung wird deutlich, dass heute knapp sieben Prozent der deutschen Bevölkerung das Doppelte bis Dreifache des durchschnittlichen Einkommens erzielen und deshalb als wohlhabend eingestuft werden (BMAS, 2013). Neben der zunehmenden sozialen Ungleichheit als Herausforderung für Politik und Gesellschaft bedeutet dies auch, dass der zunehmende Reichtum eines kleinen Teils der Bevölkerung neues Potenzial für ein vermehrtes Schenken und Stiften (Lauterbach et al., 2014:10ff) bietet. Dieser Aspekt stellt somit einen ersten wesentlichen Anknüpfungspunkt für unsere Beschäftigung mit dem Phänomen des Mäzenatentums dar.

Eine zweite wesentliche Veränderung ergibt sich in der Aufgabenverteilung zwischen Staat, Märkten und ziviler Gesellschaft (Selle, 2005:43; Heinz, 2015:17ff). Damit geht ein
„Prozess der Entstaatlichung“ (Selle, 2005:36) einher, der im internationalen Kontext unter dem Begriff der Postdemokratie diskutiert wird (Crouch, 2008). Aus der Sorge heraus, dass ein einseitig dominanter Einfluss mächtiger ökonomischer Akteure nicht angemessen legitimiert wird, werden Demokratiedefizite formuliert (Heinz, 2015:28; Belina et al., 2013:126). In einer differenzierteren Betrachtung ist aber festzuhalten, dass Stadtentwicklung - nicht erst heute - als Resultat des Handelns der Akteure aus Staat, Markt und Zivilgesellschaft gewertet wird (Selle, 2014:77ff), wenngleich sich in den vergangenen Jahrzehnten das Kräfteverhältnis zwischen öffentlicher Steuerung und privaten Interessen verschoben hat (Wiegandt, 2001:321f). Solche Verschiebungen im Gefüge der Akteure mit Veränderungen beim Steuern und Regieren der Gesellschaft werden seit einigen Jahren unter dem Stichwort der Gouvernementalität bzw. Governance (Mattissek und Prossek, 2014; Einig et al., 2005; Hohn et al., 2006) diskutiert. Die Verantwortung für gesellschaftliche Ziele verschiebt sich zwischen den Akteuren. Der Einfluss der städtischen Politik und Planung wird geringer, die Mitgestaltungsmöglichkeiten der privaten Akteure aus Wirtschaft und Zivilgesellschaft werden größer und die vormals zentrale Rolle der öffentlichen Akteure bei der Entwicklung von Städten schwindet (Selle, 2005:38). In vielen Handlungsfeldern ist der ,enabling state“ (Hohn et al., 2006:5f) nur noch ein Partner unter anderen (Fürst, 2012:34) und nicht-staatliche Akteure werden zunehmend in politischplanerische Entscheidungs- und Implementierungsprozesse eingebunden (Mattissek und Prossek, 2014:201f; Crouch, 2011:14,203ff). Dies bildet den zweiten wesentlichen Anknüpfungspunkt für unsere Beschäftigung mit dem Mäzenatentum.

Die beschriebenen Entwicklungen bleiben schließlich nicht ohne Folgen für die Stadtentwicklung. Es kommt in vielen Themenfeldern zu „Neuordnungen des Städtischen“ (Belina et al., 2013). In besonderer Weise hat sich die kritische Stadtgeographie mit diesen Entwicklungen auseinandergesetzt (Belina et al., 2014), aber auch andere Disziplinen der Stadtforschung widmen sich diesen „Neuordnungen“ (Häußermann et al., 2008; Heinz, 2015). Bei einer gleichzeitig zunehmenden Bedeutung der Städte werden öffentliche Aufgaben vom Staat auf die kommunale Ebene übertragen (Fürst, 2012). Im Spannungsverhältnis zwischen einer ,außenorientierten Wettbewerbspolitik“ und einer ,,innenorientierten Versorgungspolitik" verschieben sich in der Stadtpolitik die Gewichte auf image- und wettbewerbsorientierte Großprojekte (Heinz, 2015:158ff) mit einer Vernachlässigung ausgleichspolitischer Zielsetzungen (Fürst, 2012:34; Häußermann et al., 2008:251ff).

In der Wahrnehmung und Einordnung der gesellschaftlichen Veränderungen dominiert in der wissenschaftlichen Debatte eine eher kritische Haltung, die Mäzenatentum und andere Formen der Philanthropie als eine Facette des Abbaus sozialstaatlichen Handelns und einer schleichenden Entdemokratisierung begreift. Im Kontrast dazu steht ein breiter 
parteiübergreifender Konsens, der unterschiedliche Formen des gesellschaftlichen Engagements fördern will. Dies drückt sich u.a. in einer eigenen Enquete-Kommission zum bürgerschaftlichen Engagement aus, die die Bundesregierung Anfang der 2000er Jahre eingerichtet hatte (Deutscher Bundestag, 2002). Eine theoretische Begründung erhält ein solches politisches Handeln vor allem durch Überlegungen zur Zivilgesellschaft (z.B. Adloff, 2005; Crouch, 2011:203ff). Der „aktivierende Staat“ oder der „Gewährleistungsstaat“ werden zu politischen Leitvorstellungen, die für ein verändertes Aufgabenverständnis des Staates stehen und die die zivilgesellschaftlichen Kräfte in die gesellschaftliche Verantwortung einbinden (Fürst, 2012:30). Auch für die Aufgaben der Stadtentwicklung wird betont, dass sich die Bürger stärker in Gemeinwohlaktivitäten einbringen und Verantwortung übernehmen sollen. Moderne Gesellschaften sind so zunehmend darauf angewiesen, dass private Akteure in ihrem Handeln nicht nur private Interessen berücksichtigen, sondern zugleich auch Aspekte des Gemeinwohls beachten und sich dementsprechend engagieren sollten (z.B. Quaestio Forschung und Beratung, 2015).

\section{Begriffliche Klärungen und Entwicklung des Mäzenatentums in Deutschland}

Nach der kurzen theoretisch-konzeptionellen Einordnung ist zunächst eine Begriffsklärung erforderlich, da oft undifferenziert sowohl vom Mäzen, vom Philanthropen, vom Spender, vom Stifter als auch vom Sponsor die Rede ist. Adloff (2010:12) unterscheidet idealtypisch drei Formen des Gebens: die Philanthropie, das Spenden und das Stiften. Der Oberbegriff der Philanthropie stammt dabei aus dem angelsächsischen Kontext und wird in der Regel mit dem Begriff des Mäzenatentums ins Deutsche übersetzt.

Philanthropie bzw. Mäzenatentum werden also ,als Oberbegriffe für alle Formen des Gebens über den Familienkreis hinaus verstanden“ (Adloff, 2010:12). Als Philanthropen bzw. Mäzene werden im Weiteren zumeist wohlhabende Privatpersonen bzw. Personengruppen und die maßgeblich von ihnen getragenen, gemeinwohlorientierten Organisationen verstanden. Mit philanthropischem bzw. mäzenatischem Handeln sollte ein positiver Effekt beim Empfänger des Transfers verbunden sein. Bei gleichzeitigem Renditeverzicht des Mäzens erfolgen also keine materiellen Gegenleistungen des Begünstigten, wobei dies andere Formen der Gegenleistung an den Mäzen nicht ausschließen muss beispielsweise die persönliche Dankbarkeit des Begünstigten oder die allgemeine gesellschaftliche Anerkennung. Eine andere Form der Gegenleistung kann die Freude an einem als sinnvoll empfundenen Handeln (,,Sinnstiftung“) sein (Klein, 2010:278ff). Für mäzenatisches Handeln ist zudem entscheidend, dass das Geben bzw. Schenken von materiellen Ressourcen, aber auch von Zeit, Empathie und Kreativität auf Freiwilligkeit basiert (Strachwitz, 2009:11).
Spenden und Stiften sind zwei verschiedene mäzenatische Handlungsweisen: Spenden ist zunächst nur der „Transfer“ und nicht der „Tausch“ von Geld, Sachen und Leistungen für gemeinwohlorientierte Zwecke (Priller und Sommerfeld, 2005:9; Adloff, 2010:12f), während das Stiften einen „Akt der Institutionalisierung“ darstellt, bei dem ein Vermögen dauerhaft und zweckgebunden einem Stifterwillen gewidmet wird. In Anlehnung an die Theorie der Gabe und Reziprozität (Adloff, 2010:34ff) handelt sich also um ,organisierte Philanthropie“ (Adloff, 2010:11). Unter diesem nicht-staatlichen Akteur der Stiftungen betrachten wir im Weiteren überwiegend die Stiftungen, die von wohlhabenden Privatpersonen gegründet wurden. 2006 waren dies 68 Prozent aller Stiftungen (Adloff, 2010:115). Davon zu unterscheiden sind Stiftungen, die von Körperschaften, also Vereinen und Verbänden, Unternehmen oder öffentlichen Körperschaften gegründet wurden. Hier gibt es eine große Vielfalt, die von den politischen Stiftungen bis zu den Bürgerstiftungen reicht (Bundesverband Deutscher Stiftungen, 2010:112f).

Noch zum Ende des 20. Jahrhunderts wurde Deutschland oftmals als Entwicklungsland in Sachen Mäzenatentum dargestellt. Diese Einschätzung entstand vor allem in Kontrast zu den USA, die im Verhältnis von sozialstaatlicher und zivilgesellschaftlicher Verantwortlichkeit anders als Westeuropa und Deutschland austariert sind (Adloff, 2010:18f; Krimphove, 2011). Das Spenden und Stiften wird hier als eine wichtige humanitäre Aufgabe angesehen, die weit verbreitet ist und im Gegensatz zu Deutschland eine hohe gesellschaftliche Wertschätzung genießt (Ostrower, 1995:36ff). Das angelsächsische Stiftungsmodell unterscheidet sich grundlegend vom traditionellen Stiftungswesen europäischer Prägung, sodass eine unmittelbare Übertragbarkeit nicht empfohlen wird (Müller-Jentsch, 2014:23ff; 59).

In Deutschland haben das Mäzenatentum und das damit verbundene Stiftungswesen eine lange, bis ins Mittelalter zurückreichende Tradition. Viele Einrichtungen, die heute selbstverständlich dem öffentlichen Sektor zugeordnet werden (Museen, Opernhäuser, Universitäten, Bibliotheken etc.), haben ihren Ursprung im mäzenatischen Engagement. Diese Traditionslinien wurden in Deutschland in der ersten Hälfte des 20. Jahrhunderts unterbrochen (Faller, 2011:39). Während in Westdeutschland nach 1945 eine langsame Wiederbelebung möglich war, hat das SED-Regime eine derartige Entwicklung in der DDR nicht zugelassen.

Zunächst blieb jedoch auch in der alten Bundesrepublik die Zahl der Stiftungsgründungen gering. Nach Angaben des Bundesverbandes deutscher Stiftungen wurden erstmals 1983 mehr als 100 Stiftungen in einem Jahr neu errichtet. Erst in den letzten zehn bis 20 Jahren hat das Stiftungswesen in Deutschland wieder an Bedeutung gewonnen. Im Durchschnitt der Jahre 2000 bis 2013 waren es nach der Wiedervereinigung 850 Neugründungen pro Jahr, die auch durch die Reformen des Stiftungswesens 2000/2002 und 2007 begünstigt wurden. Wichtiger als die gesetzlichen Änderungen dürften jedoch die langfristige wirtschaftliche und gesellschaftli- 
che Entwicklung und mit ihr die veränderte Rolle des Wohlfahrtsstaates gewesen sein (vgl. Abschnitt 2).

\section{Zum methodischen Vorgehen}

Zur Beantwortung unserer Forschungsfragen haben wir vier unterschiedliche methodische Zugänge verfolgt:

- Um den Stellenwert des Mäzenatentums für die deutsche Stadtentwicklung zu erfassen, wurde eine bundesweite Umfrage mit einem standardisierten Fragebogen unter allen 82 deutschen Großstädten mit mehr als 100.000 Einwohnern durchgeführt. Ergänzt wurde diese Umfrage durch eine Befragung aller 396 Städte und Gemeinden in Nordrhein-Westfalen.

- Einzelne Projekte in Hamburg, Wuppertal und Potsdam wurden in Form von qualitativen Expertengesprächen sowie einer umfangreichen Medienanalyse untersucht. Hierfür wurde auf die Kontakte und Erkenntnisse der Städtebefragung zurückgegriffen. Zudem wurden Interviews mit Experten geführt, die das gesamte mäzenatische Engagement in einer Stadt überblicken. Um stadtspezifische Unterschiede herausarbeiten zu können, wurden hierfür neben Hamburg, Wuppertal und Potsdam die vier Städte Bielefeld, Gütersloh, Heidelberg und Münster hinzugezogen. Insgesamt wurden 24 qualitative Experteninterviews geführt.

- Als ein Schwerpunkt des Forschungsinteresses stellte sich die Frage nach den Motiven des mäzenatischen Handelns auf lokaler Ebene heraus. Dabei rückten Stiftungen als institutionalisierte Form des Mäzenatentums in den Fokus unseres Interesses. Mäzene handeln überwiegend in dieser institutionalisierten Form. Um unsere Ergebnisse auf eine breite Datengrundlage zu stellen, wurden in einer bundesweiten schriftlichen Befragung 5.080 Stiftungen in 22 deutschen Großstädten angeschrieben. 900 Fragebögen wurden beantwortet.

- Die Ergebnisse dieser Umfrage waren zugleich auch die Vorbereitung auf unseren letzten empirischen Arbeitsschritt. In 16 weiteren qualitativen Interviews wurden gezielt Stifterpersönlichkeiten und Stiftungsverwalter angesprochen, um die Motive der Stiftungsgründungen, die Stiftungsarbeit und vor allem das Verhältnis der Stifter zu ihrer Stadt genauer zu hinterfragen.

\section{Ungleichgewichte im Stellenwert des Mäzenatentums zwischen deutschen Städten}

In diesem Abschnitt wollen wir die Rolle von stadtspezifischen Gegebenheiten und Bedingungen für das lokale Mäzenatentum klären. Wir werden einige Gründe benennen, wieso

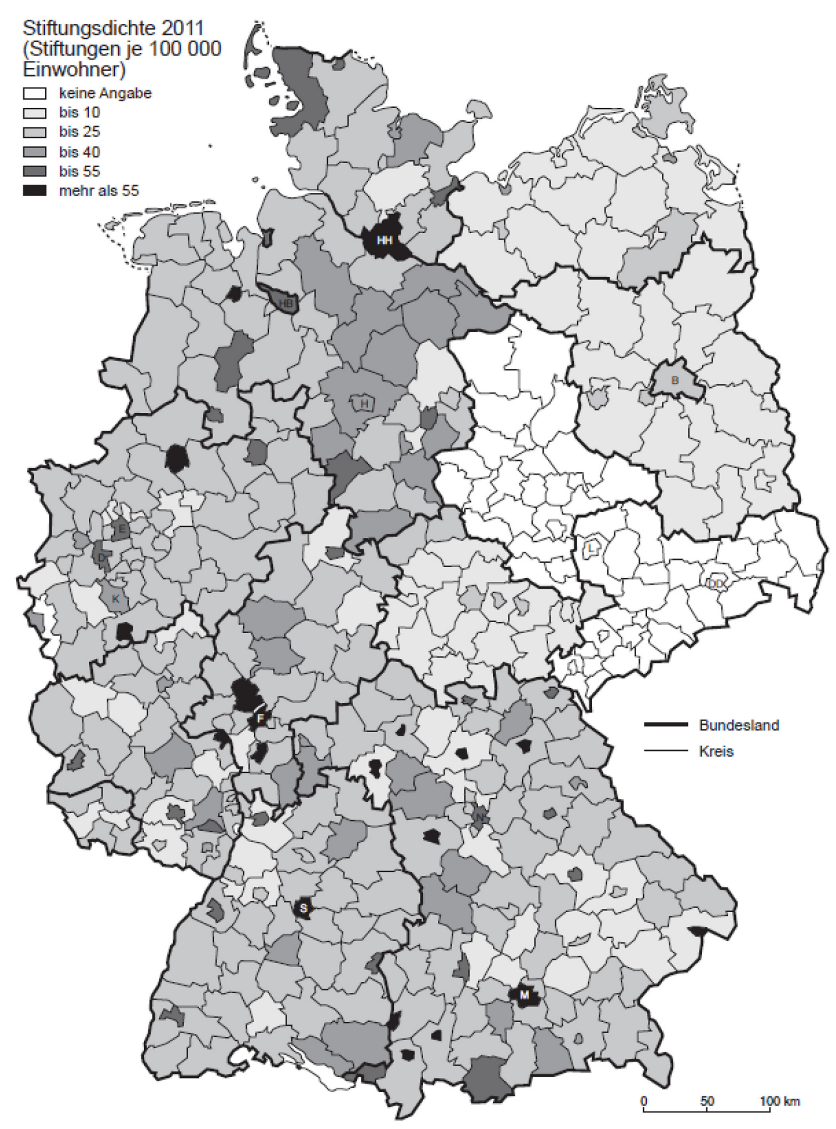

Abb. 1. Räumliche Verteilung der Stiftungen in Deutschland (im Jahr 2011).

mäzenatisches Handeln einen jeweils unterschiedlichen Stellenwert in deutschen Städten hat und wie es dadurch zu unterschiedlichen Verteilungsmustern des mäzenatischen Handelns in Deutschland kommt (vgl. Abb. 1 und Bundesverband Deutscher Stiftungen, 2010:94ff). Eine erste offensichtliche Ursache liegt in der ungleichen räumlichen Verteilung von Wohlstand und Reichtum in Deutschland. Allerdings dürften die räumlichen Ungleichgewichte einer daraus resultierenden Elitephilanthropie nicht ganz so ausgeprägt sein, wie ein heuristisch angelegter regionaler Vergleich der Wirtschaftskraft und der wirtschaftlichen Entwicklungsdynamik vermuten lässt. Denn im heutigen räumlichen Muster der Philanthropie spiegeln sich auch die regionalen Muster früherer Wirtschaftszyklen und die daraus entstandenen ,alten Vermögen“.

Historisch bedingte Unterschiede wirken also bis heute nach. Sehr offensichtlich ist dies zunächst im Vergleich von westdeutschen und ostdeutschen Städten. In Ostdeutschland ist die Stiftungsdichte in einem $\mathrm{Ma}$ geringer, das sich kaum allein durch ökonomische Unterschiede erklären lässt. Ein bürgerschaftliches Engagement konnte sich im DDR-Staat kaum entfalten. Dies hat auch heute noch vielerorts Konse- 
quenzen in der meist geringeren Vitalität des mäzenatischen Handelns.

Auch wenn die Unterschiede zwischen den westdeutschen Regionen bzw. Städten bei weitem nicht so prägnant sind wie die Unterschiede zwischen Ost- und Westdeutschland, verfügen die ehemaligen „Arbeiterstädte“ des Ruhrgebietes auch heute meist noch über eine weniger ausgeprägte Bürgerkultur als die früheren süddeutschen „Bürgerstädte“. Mit Blick auf die historischen Wurzeln der deutschen Städte lassen sich stark vereinfacht folgende vier Stadttypen voneinander abgrenzen:

\subsection{Die ehemalige Handwerker-, Handels- und Bürgerstadt}

Dieser erste Stadttyp repräsentiert die europäische Stadt in besonderer Weise. In diesen früher freien Städten haben ständische Organisationen und Honoratioren das Stadtleben wesentlich geprägt. Dadurch konnte sich eine bürgerliche Gesinnung und Verantwortungskultur etablieren, die bis heute als Triebfeder für bürgerschaftliches Engagement und mäzenatisches Handeln nachwirkt. Zumindest wird diese Argumentationslinie in den von uns näher betrachteten Städten häufig genutzt. Demnach gibt es eine ungeschriebene Verpflichtung für erfolgreiche Bürger, der Stadt etwas zurückzugeben, in der sie es zu etwas gebracht haben. Dies gilt auch für die zugezogenen Bürger, die sich mit ihren lokal wirksamen Spenden Anerkennung in der traditionellen Stadtgesellschaft verschaffen wollen.

\subsection{Die klassische Industrie- und Arbeiterstadt}

Jüngere Stadtgründungen des 19. Jahrhunderts und frühen 20. Jahrhunderts haben keine vergleichbaren Traditionen. Die Stadtwerdung ist hier ein Ergebnis der Industrialisierung. Die politische Kultur ist in besonderer Weise mit der Arbeiterbewegung und später mit der sozialdemokratischen Vorstellung eines starken Staates verbunden, der weite Teile des gesellschaftlichen Lebens regelt. Individuelle bürgerliche Verantwortungsübernahme - so kann man vermuten - ist hier weniger ausgeprägt.

\subsection{Die frühe Manufaktur- und Industriestadt}

Sie unterscheidet sich vom vorhergehenden Stadttyp durch eine frühere Industrialisierung, die noch stärker in der handwerklichen Tradition verwurzelt ist. Zu denken ist z.B. an die mit der Textilmanufaktur verbundenen Städte Krefeld oder Mönchengladbach. Auch Wuppertal ist hier - vor einem etwas anders gelagerten industriegeschichtlichen Hintergrund - einzuordnen. Prägend für diesen Stadttyp ist ebenso, dass es bereits im 19. Jahrhundert zu krisenhaften Entwicklungen mit einer hohen Arbeitslosigkeit kam, ohne dass bereits ein leistungsfähiger Sozialstaat vorhanden war, der diese Probleme abgefedert hätte. Die Notwendigkeit zur Armutsbekämp- fung hat hier bürgerschaftliche Tugenden und mäzenatisches Handeln gefordert und ggf. bis heute wirksame Traditionslinien begründet.

\subsection{Die einstige Fürsten- und Verwaltungsstadt}

Die Besonderheit dieses Stadttyps besteht in der dauerhaften politisch-administrativen Funktion dieser Städte. In diesem Kontext wird ein bürgerliches Mäzenatentum beschrieben, das im Wesentlichen darauf gerichtet ist, es dem Fürsten gleich zu tun und hierüber Zugang zum Fürstenhof zu erhalten. Zwar kann man vermuten, dass auch dieses Mäzenatentum Traditionslinien begründet, doch werden diese vor Ort kaum als Bezugspunkt eines aktuellen Mäzenatentums angesprochen. Beispielstädte sind Bonn, Münster, Mannheim oder Würzburg.

Die historischen Wurzeln mögen als Traditionslinien im „kollektiven Gedächtnis“ der einzelnen Städte verankert sein, wurden jedoch mit der Ausformung relativ einheitlicher und durchgreifender sozialstaatlicher Strukturen zunehmend überdeckt. Wenn sich heute Unterschiede in der Dynamik des philanthropischen Sektors zwischen den Städten zeigen, hat dies neben den historischen Wurzeln auch mit der jüngeren regionalen Wirtschaftsgeschichte $\mathrm{zu}$ tun. $\mathrm{Zu}$ denken ist dabei an Bertelsmann in Gütersloh oder Oetker in Bielefeld, Otto und einige andere Mäzene in Hamburg sowie die SAPGründer (Hopp, Tschira, Plattner) in Walldorf und Heidelberg. Nicht zuletzt müssen die Unterschiede beim mäzenatischen Handeln auch im Kontext einer kommunal aktiv geförderten Engagement- und Anerkennungskultur gesehen werden. Unsere Recherchen zeigen, dass in diesem Sinne aktive Städte über eine lebendigere und gegenüber der Kommune relativ positiv eingestellte philanthropische Szene verfügen. Dies gilt etwa für Nürnberg oder Stuttgart.

\section{Bedeutung und Umfang des Iokalen mäzenatischen Engagements}

Auf der Basis unserer Stiftungsbefragung lassen sich die Strukturen und der Umfang des lokalen Engagements des Stiftungssektors abschätzen. Die Kurzbefragung der Kommunen liefert zudem Einschätzungen zur Bedeutung des lokalen Mäzenatentums in den Städten.

Unsere Städtebefragung hat zunächst die vorliegende Einschätzung bestätigt, dass insbesondere die Großstädte Orte eines lokalen philanthropischen Engagements sind. Von den 396 befragten nordrhein-westfälischen Städten geben 40 Prozent an, dass bei ihnen Mäzenatentum eine Rolle spielt (Rücklauf $38 \%=151$ ). Dabei sieht knapp die Hälfte dieser Städte $(43 \%)$ eine historische Kontinuität. In den bundesweit befragten Großstädten mit mehr als 100.000 Einwohnern erkennen 87 Prozent eine Bedeutung des lokalen Mäzenatentums (Rücklauf $57 \%$ ), wovon wiederum 80 Prozent sowohl eine historische als auch eine aktuelle Bedeutung sehen. Immerhin 38 Prozent der Großstädte ordnen den mit dem Mäze- 


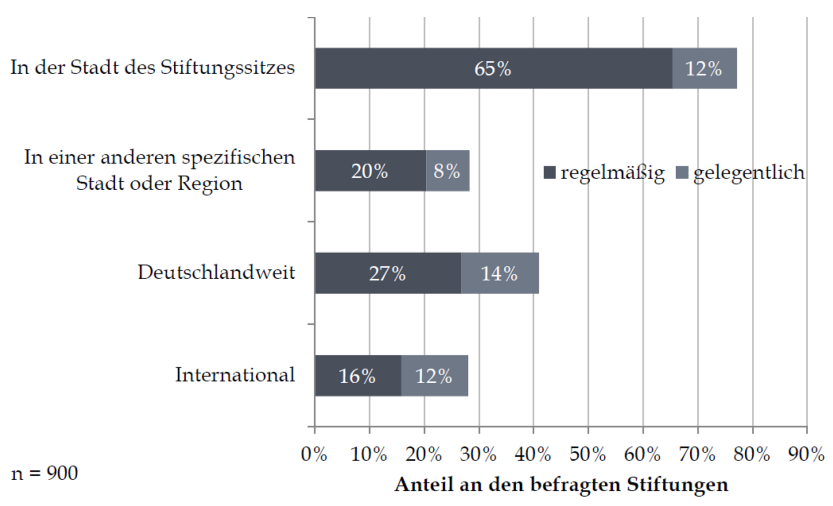

Abb. 2. Intensität und räumlicher Bezug des mäzenatischen Engagements.

natentum verbundenen Projekten eine große Bedeutung für die Stadtentwicklung zu (41\% eine mittlere und $21 \%$ eine geringe Bedeutung).

In unserer Stiftungsbefragung wurden rund 5.000 Stiftungen angeschrieben, von denen 904 bzw. 17,8 Prozent teilgenommen haben. Dabei zeigt sich, dass der Stiftungssektor in einem hohen Maße lokal ausgerichtet ist. 65 Prozent der Stiftungen geben an, sich regelmäßig und zusätzliche 12 Prozent sich gelegentlich in der Stadt des Stiftungssitzes zu engagieren. So sind insgesamt 77 Prozent der Stiftungen vor Ort aktiv. Das überregionale/deutschlandweite oder gar internationale Engagement fällt demgegenüber deutlich zurück (vgl. Abb. 2).

In der öffentlichen Wahrnehmung wird die wirtschaftliche Leistungsfähigkeit des Stiftungssektors überschätzt. Eine Ursache wird sein, dass vor allem die sehr leistungsfähigen, oft unternehmensverbundenen Stiftungen mit ihren Projekten und vielfach auch politisch relevanten Ergebnissen in den Medien präsent sind. Tatsächlich wird die Stiftungslandschaft quantitativ aber von eher kleinen, weniger leistungsfähigen Stiftungen dominiert. Dies gilt für den Stiftungssektor insgesamt und insbesondere für die lokal engagierten Stiftungen. Bei knapp 22 Prozent aller befragten Stiftungen liegt das jährliche, lokal wirksame Fördervolumen unterhalb von $5.000 €$ (vgl. Abb. 3). Nimmt man die Stiftungen mit einem lokalen Fördervolumen von bis zu $50.000 €$ hinzu, sind damit 60 Prozent aller antwortenden Stiftungen erfasst. Immerhin bewegen sich 16 Prozent mit ihrem Fördervolumen in einem Bereich zwischen 50.000 und 250.000 $€$ und weitere 6,5 Prozent noch darüber. Unsere Erhebungen zeigen auch, dass 42 Prozent der Stiftungen ausschließlich lokal aktiv sind bzw. ihr gesamtes Fördervolumen lokal einsetzen. Im Mittel der befragten Stiftungen beträgt der lokale Ausgabenanteil 62 Prozent und das lokale Fördervolumen jährlich knapp $95.000 €$.

Damit liefern unsere Ergebnisse Anhaltspunkte für eine grobe Abschätzung der wirtschaftlichen Bedeutung des lokalen Stiftungsengagements. Rechnet man den von uns ermit-

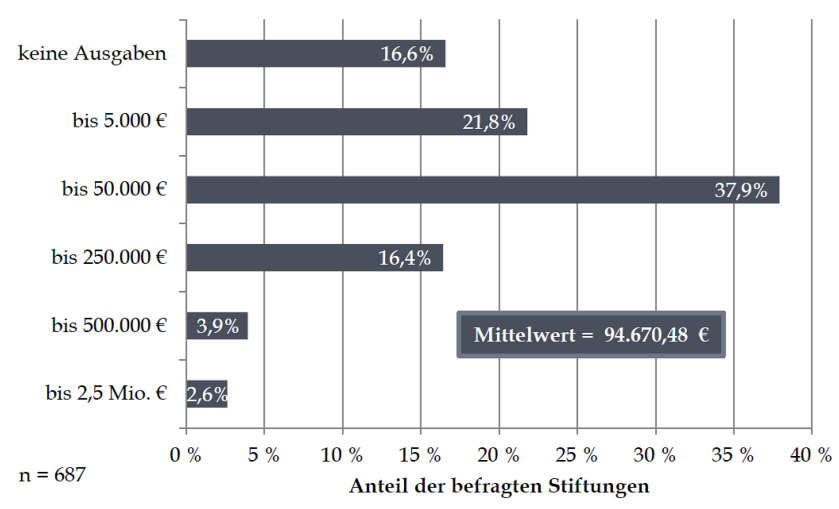

Abb. 3. Lokal wirksames Fördervolumen.

telten Anteil lokal engagierter Stiftungen (77\%) auf den Gesamtbestand der im Verzeichnis des Bundesverbandes deutscher Stiftungen erfassten Stiftungen hoch (knapp 20.000 zum Jahresende 2012) und unterstellt ein lokales Fördervolumen in der von uns ermittelten Höhe, ergibt sich ein Gesamtwert des jährlichen lokalen Engagements von über 1,5 Milliarden $€$.

\section{Motive und Hintergründe des lokalen mäzenatischen Engagements}

Die Motive des philanthropischen Engagements sind vielfältig. Insbesondere Timmer (2006:23ff) hat sich empirisch mit den Beweggründen von Stiftern auseinandergesetzt, sich finanziell zu engagieren. Der „Wunsch, etwas zu bewegen“ bzw. ,ein konkretes Problem zu bekämpfen“ ist dabei ein besonders ausgeprägtes Motiv. Nahezu ebenso häufig wird von Stiftern eine positive Haltung gegenüber der Gesellschaft artikuliert, die sowohl als eine ,moralische Verpflichtung“ als auch als ,Dankbarkeit“ und ein damit verbundenes ,Zurückgeben an die Gesellschaft" empfunden werden kann. Empathie drückt sich als Motiv insbesondere in einem,,Verantwortungsbewusstsein gegenüber Mitmenschen“ und dem ,Mitleid mit Notleidenden“ aus. Schließlich spielen auch ,,religiöse Überzeugungen“, das „,Andenken an nahestehende Personen“, die „Familientradition“ oder auch das „Fortführen beruflicher Aktivitäten in einem anderen Rahmen“ eine Rolle.

Wir erwarten nicht, dass diese Motive und Beweggründe bei einem primär lokal ausgerichteten Engagement anders gelagert sind. Wichtig ist jedoch die Erkenntnis, dass mäzenatisches Geben weniger aus inneren Beweggründen als aus sozialen Einbettungen heraus beflügelt wird: „Nicht der einzelne, der einsam seine altruistischen Entscheidungen trifft, konstituiert Philanthropie, sondern in Gruppenprozessen des Gebens und Nehmens werden auf der Mikroebene bürgerschaftliche Identitäten kreiert, die bis in größere Zusammenhänge ausstrahlen können“ (Adloff, 2010:63). Diese Gruppenprozesse und die mit ihnen verbundenen sozialen Bezie- 
hungen können dabei mehr oder weniger im lokalen und damit im institutionellen Umfeld der Stadt verankert sein. Die Intensität und Qualität dieser lokalen Einbettung könnte insofern auch ein Prädiktor für lokales mäzenatisches Engagement sein.

Unsere Stiftungsbefragung lässt derartige Zusammenhänge erkennen. So ist die Wahrscheinlichkeit für ein lokales Engagement mehr als doppelt so hoch $(70 \%$ gegenüber $30 \%$ bei geringer Bindung an die Stadt), wenn die Befragten angeben, dass der Stifter eine hohe Bindung an die Stadt hatte oder hat. Dabei ist kaum von Bedeutung, aus welchen biografischen Abschnitten (Kindheit, Ausbildung, Berufsleben) dies resultiert. Besonders häufig resultiert die Bindung aus der Phase des Berufslebens, da die Stiftungsgründung überwiegend im letzten Abschnitt oder am Ende des Berufslebens erfolgt. In unseren Gesprächen mit Mäzenen wurde aber auch deutlich, dass die Orte von Kindheit und Ausbildung oft nicht in Vergessenheit geraten sind und ein Teil des Engagements dorthin gelenkt wird.

Die hohe Bedeutung der sozialen Einbindung vor Ort, die sich in den vorgenannten Ergebnissen zeigt, findet sich ebenfalls in unseren Gesprächen mit Experten und Mäzenen wieder. Vielfach wird beschrieben, wie das Engagement eines einzelnen Mäzens zum Ausgangspunkt wird, weitere Mäzene einzubeziehen. Die erste Unterstützung hat eine Initialfunktion zur Ansprache und Einwerbung weiterer Gaben. Der stadtbekannte Großmäzen wird hier zum Kristallisationspunkt eines Gruppenbildungsprozesses, wobei man zwei Wirkmechanismen vermuten kann. Die Erstunterstützung kann man erstens als Auszeichnung für das Projekt verstehen, die weiteren Mäzenen eine Beteiligung erleichtert. Zweitens können sich nachträglich angesprochene Mäzene durch ihre Unterstützung auch sozial in die Nähe des ersten Großspenders rücken.

Die philanthropische Szene in den von uns betrachteten Städten lässt sich kaum als ein in den Alltag hineinwirkendes enges persönliches Geflecht zwischen den Mäzenen beschreiben. Es handelt sich mehr um ein ,,voneinander wissen“ und ein „Beobachten aus der Distanz“. Enge persönliche Kontakte zwischen den Mäzenen sind zwar nicht ausgeschlossen, nach unseren Erhebungen aber kaum konstituierend für das philanthropische Netzwerk. Aber auch diese distanzierte Beobachtung dürfte eine Wirkung auf die Stabilisierung und Förderung des philanthropischen Handelns in der Stadt haben, weil sich damit auch eine stillschweigende Verständigung auf bestimmte Werte (z.B. Haltung gegenüber der Stadtgesellschaft) und damit verbundene Handlungsweisen verbinden kann. Das Zusammentreffen in elitären Clubs (Lions, Rotary usw.) unterstützt zumindest in Teilsegmenten der philanthropischen Szene die gegenseitige Selbstbeobachtung sowie auch die Selbstvergewisserung der Zugehörigkeit zur städtischen Elite.

Durchgängig wird sowohl von den kommunalen Experten als auch von den befragten Mäzenen die hohe Bedeutung der persönlichen Kontakte zwischen politischer Spitze und
Mäzenen beschrieben. Dabei wird einerseits Wertschätzung und Anerkennung auch als Antriebsmoment für das gesellschaftliche Engagement vermittelt. Gerade der im Grenzbereich zu öffentlichen Aufgaben agierende Mäzen hat oftmals nicht nur den persönlichen Anspruch auf ein ,gleichrangiges“ Gegenüber, sondern auch ein Interesse, dass sein Projekt bei der öffentlichen Hand zur „Chefsache“ und somit in verlässliche und vertrauliche Kooperationsbeziehungen eingebettet wird. Dies dient nicht nur oder primär der Eitelkeit, sondern entsteht auch aus dem Wunsch nach kalkulierbaren Umsetzungsbedingungen.

\section{Die thematische Ausrichtung des lokalen mäzenatischen Engagements}

Stiftungen engagieren sich lokal in verschiedenen Handlungsfeldern. Insgesamt dominieren „soziale Zwecke“ sowie „Bildung und Erziehung“ das thematische Portfolio (vgl. Abb. 4). Im Ranking der lokalen Engagementbereiche folgen „Kunst und Kultur“ sowie „Wissenschaft und Forschung“. Die Themen „Denkmalschutz/öffentlicher Raum“, „Bürgerengagement/politische Teilhabe“ sowie „Umweltschutz" fallen demgegenüber in ihrer Bedeutung deutlich zurück.

Das Zustandekommen und die Weiterentwicklung des philanthropischen Engagements hat viel mit der sozialen Einbindung in die Stadt zu tun. Unabhängig davon bleibt die thematische Grundausrichtung im Individuellen verhaftet. Unsere vertiefenden Gespräche mit Stiftern und Stiftungen zeigen vielfältige biografische Bezüge. Persönliche Vorlieben für bestimmte Kunstsparten, Schicksalsschläge wie Krankheiten im familiären Umfeld oder häufiger noch die im beruflichen Umfeld gesammelten Erfahrungen prägen die thematische Ausrichtung des philanthropischen Engagements. Fast idealtypisch finden sich diese unterschiedlichen biografischen Bezüge im Engagement der Wuppertaler Jackstädt-Stiftung. Das kinderlose Ehepaar hat sich nach dem Verkauf des in Wuppertal aufgebauten Familienunternehmens auf drei Themen für ihr finanzielles Engagement konzentriert. Die Unternehmerbiografie war Anlass zur Unterstützung der betriebswirtschaftlichen Forschung, die Erblindung in Folge einer bislang nicht behandelbaren Augenerkrankung führte zur Herausbildung eines entsprechenden ophthalmologischen Förderschwerpunktes und schließlich hat die mit der unternehmerischen Tätigkeit gewachsene Bindung an den Standort Wuppertal den Ausschlag für einen lokalen Förderschwerpunkt gegeben. Charakteristisch für das lokale Engagement der Stiftung sind dabei weniger thematische Schwerpunkte als vielmehr der Wunsch, der ehemals stolzen und heute wirtschaftlich und finanziell darbenden Stadt zu einigen Glanzpunkten (,,i-Tüpfelchen“) zu verhelfen. So wurden z.B. im Rahmen der Sanierung der Oper eine hochwertigere Ausstattung finanziert, eine Starthilfe für die Reaktivierung einer stillgelegten Bahntrasse als 


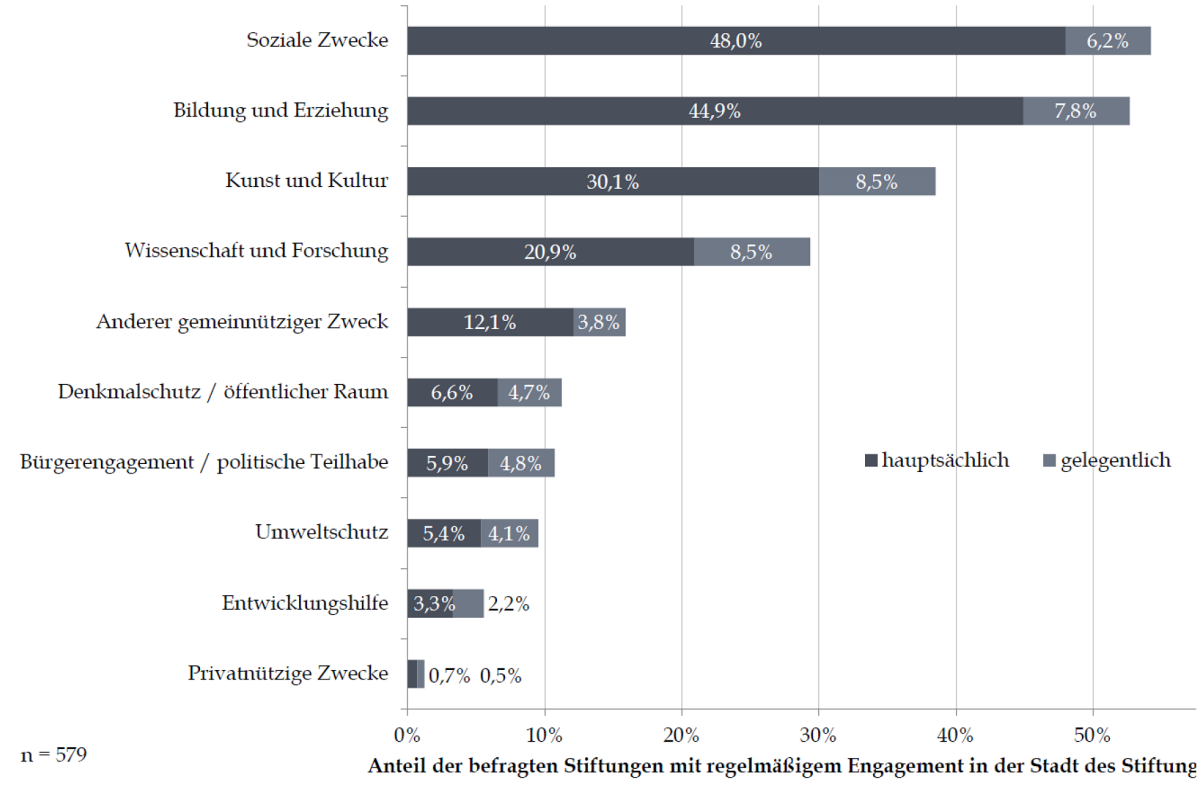

Abb. 4. Handlungsfelder des lokalen mäzenatischen Engagements.

Rad- und Wanderweg gegeben („Nordbahntrasse“) und nicht zuletzt auch die Realisierung der ,Junior-Universität“ unterstützt.

Als Gründe für die lokale Ausrichtung geben mit $46 \%$ fast die Hälfte der befragten Stiftungen ,biografische Bezüge" des Stifters als wichtig bzw. ausschlaggebend an. Oftmals ist es auch die Wertschätzung einer spezifischen lokalen Einrichtung, die zu ihrer gezielten Förderung geführt hat (34\%). Einige Stiftungen geben an, dass sich ihre Ausrichtung im Verlauf der Stiftungsarbeit herauskristallisiert und gefestigt hat $(16 \%)$. Hier kann man vermuten, dass zunächst weniger eindeutige thematische Präferenzen vorgelegen haben, die Stiftungen stattdessen stärker im Dialog mit lokalen Akteuren stehen und sich hieraus ihre Ausrichtung entwickelt hat. Schließlich agieren 14 Prozent der Stiftungen aus einer „Unzufriedenheit mit der Leistungsfähigkeit des örtlichen Gemeinwesens" heraus, was sich überproportional häufig mit einer Fokussierung auf das Thema „Bürgerengagement/politische Teilhabe" verbindet.

Konzeptionelle Überlegungen der Stiftungsarbeit spielen bei Förder- und Vergabeentscheidungen eine geringere Rolle. Solche Entscheidungen basieren mehr auf der persönlichen Wertschätzung der Partner und Empfänger als auf einer kritischen Auseinandersetzung mit dem geförderten Projekt. So beschreiben die Mitarbeiter einer bedeutenden Hamburger Stiftung ihre Vergabeentscheidungen als sehr stark vom Stifter und dessen persönlicher Einschätzung („,Bauchgefühl“) heraus gesteuert. Ein Wuppertaler Museumsleiter macht die hohe Bedeutung der persönlichen Überzeugung folgendermaßen deutlich: „Sie müssen dem Mäzen schon mit Schweißperlen auf der Stirn entgegentreten, die erkennen lassen, dass Sie ihre Sache ernst meinen."
Die stark durch persönliche Einschätzungen gesteuerten Förderentscheidungen sind schwer oder kaum objektivierbar. Sie sind somit zugleich Ausdruck des persönlichen Gestaltungsspielraums des Stifters bzw. der Stiftungen bei der Verwendung der Mittel und der Steuerung der Stiftungsarbeit. Es ist naheliegend und hat sich in unseren Gesprächen bestätigt, dass nach diesem Muster agierende Philanthropen und Stiftungen die Einbettung ihrer Förderaktivitäten in öffentliche oder politische Diskurse meiden. Somit werden Projekte und Kooperationen im Umfeld politisch relevanter Themen und Maßnahmen gemieden. Denn die Kooperation mit Akteuren aus dem politisch-administrativen Bereich und die Einbettung in politische Diskurse bergen das Risiko, Relevanz- und Wirksamkeitsargumente zur Rechtfertigung der Förderaktivitäten liefern zu müssen. Dies kann die Freiheit in der Mittelverwendung insbesondere bei kleineren Stiftungen beschränken, bei denen der Stifter als Person ein hohes Gewicht bei den Vergabeentscheidungen hat.

\section{Vier Typen mäzenatischer Projekte aus kommunaler Governanceperspektive}

Von besonderem Interesse ist für uns ein Mäzenatentum, das auf der Schnittstelle zum kommunalen Handeln angesiedelt ist. Für eine Systematisierung des Verhältnisses zwischen Kommune und Mäzen lassen sich auf der Basis unserer Interviews idealtypisch vier Projekttypen definieren. Die Reihenfolge der drei ersten Typen steht auch für eine ansteigende Intensität möglicher Interessenkonflikte und damit zugleich der erforderlichen Aushandlungsprozesse zwischen Kommune und Mäzen. 
9.1 Typ 1 - autonome Projekte mit Genehmigungsvoraussetzungen

Ein Mäzen realisiert in einem dominant privatrechtlichen Rahmen ein Projekt (z.B. Aufbau und Betrieb einer Sozialstation mit Leistungsangeboten für eine bestimmte Zielgruppe oder ein bestimmtes Stadtquartier). Sofern hierzu eine öffentliche Genehmigung für den Bau oder Betrieb einer Einrichtung erforderlich ist, kann die öffentliche Hand dies wie einen normalen Genehmigungsvorgang behandeln. Ein Interessenskonflikt kann entstehen, wenn im Genehmigungsverfahren Ermessensspielräume bestehen, die der Mäzen unter Verweis auf das besondere, im öffentlichen Interesse stehende Engagement zu seinen Gunsten genutzt sehen möchte.

Ein Beispiel ist etwa die Gründung des „Hauses der Frauengeschichte (HDFG)“ in Bonn, das im Jahr 2012 eröffnet wurde. Eine emeritierte Professsorin hatte ihr berufliches Engagement für die Frauengeschichtsforschung zunächst in einen entsprechenden Förderverein verlagert und die Gründung des HDFG vorbereitet. Die später gegründete „AnnetteKuhn-Stiftung" fungiert als Trägerorganisation des HDFG. Die erforderlichen baurechtlichen Genehmigungen und die Gründung der Stiftung sind dabei weitgehend unproblematisch verlaufen. Allerdings bestanden auf Seiten der Stifterin Erwartungen gegenüber dem Land und der Kommune auf eine weitergehende finanzielle Unterstuitzung, die jedoch nicht erfüllt wurden.

\subsection{Typ 2 - Kofinanzierungsprojekte}

Ein Mäzen finanziert eine bestehende öffentliche Einrichtung (z.B. die Kofinanzierung einer von Schließung bedrohten Stadtteilbibliothek, eines Kindergartens oder einer Schule). Mit der Kofinanzierung können mehr oder weniger starke Vorstellungen zur Mittelverwendung verbunden sein sowie auch unterschiedlich intensive Versuche, die Mittelverwendung zu beeinflussen. Die Vorstellungen der Mäzene können in Konflikt zu den öffentlichen Vorstellungen (z.B. Betriebsrichtlinien oder Lehrplan, Präferenz zur Renovierung von Räumlichkeiten gegenüber Präferenz zur Anschaffung von Lernmitteln) stehen. Kofinanzierungsprojekte lösen keine zusätzlichen komplementären öffentlichen Mittel aus. Mit Blick auf die Gesamtkosten aus Investition und Betrieb entsteht so eine Teilfinanzierung des jeweiligen Projekts. Die Initiative zur Realisierung der Einrichtung sowie die programmatische Grundkonzeption für Aufbau und Betrieb gehen von der öffentlichen Hand aus. Es handelt sich also um ein öffentliches Projekt, das privat kofinanziert wird, wobei dies auch durch geldwerte Leistungen (z.B. Arbeitsstunden) des Mäzens erbracht werden kann. Kofinanzierungsprojekte können sowohl den Charakter von Betriebskosten- als auch den Charakter von Investitionskostenzuschüssen bis hin zur Übernahme der kompletten Investitionskosten annehmen.

Die bereits erwähnte Finanzierung einer hochwertigen Ausstattung (Bestuhlung) bei der Sanierung der Wupperta- ler Oper ist ein solches Kofinanzierungsprojekt. Probleme können entstehen, weil die privaten Teilprojekte in das öffentliche Vorhaben integriert (inkl. Ausschreibung) und von daher der privaten Steuerung entzogen werden. Dies setzt ein besonderes Vertrauensverhältnis und eine grundlegende Akzeptanz vom Mäzen gegenüber der öffentlichen Hand und den dort üblichen Handlungsweisen voraus. Was im kleinen Rahmen in Wuppertal auf der Basis einer vertraulichen $\mathrm{Zu}-$ sammenarbeit zwischen Mäzen und Stadtspitze gelungen ist, hat in Hamburg bei den Großprojekten des Ehepaars Greve zu erheblichen Kontroversen mit bundesweitem Medienecho geführt. Das Ehepaar Greve hatte sich Mitte der 1990er Jahre an den Regierenden Bürgermeister gewandt und ein größeres Geschenk aus Anlass des 75jährigen Uni-Jubiläums in Aussicht gestellt. Nach entsprechenden Vorüberlegungen mit der Universität wurde eine Erweiterung des Universitätshauptgebäudes vorgeschlagen. Das Ehepaar Greve hatte eingewilligt und sich schließlich in einer Allianz mit Stadt- und Universitätsspitze und gegen den Widerstand weiter Teile der an Architektur- und Städtebaufragen interessierten Fachöffentlichkeit mit der Absicht durchgesetzt, die „Flügelbauten“ mit einem eigenen Architekten und ohne ein übliches Wettbewerbsverfahren zu realisieren (Faller und Wiegandt, 2015). Unsere Interviews mit ausgewählten Beteiligten ließen erkennen, dass die damit verbundenen Auseinandersetzungen nicht nur bis heute nachwirkende persönliche Verletzungen erzeugten, sondern auch einem in Hamburg ansonsten weniger verbreiteten Misstrauen gegenüber Mäzenen Vorschub geleistet haben.

\subsection{Typ 3 - Komplementärprojekte}

Beim Komplementärprojekt liegt die Initiative entweder beim Mäzen selbst oder gleichberechtigt beim Mäzen und der öffentlichen Hand. Die Grundkonzeption wird dementsprechend zum Gegenstand einer Aushandlung. Durch das mäzenatische Geschenk wird ein Impuls zur Mobilisierung komplementärer öffentlicher Mittel ausgelöst, der bei der öffentlichen Hand zu Überlegungen und Entscheidungen der alternativen Verwendungsmöglichkeit führt. Ein typisches Beispiel wäre es, wenn ein Mäzen der Stadt eine Kunstsammlung vermacht, sodass hier Überlegungen zu deren Unterbringung und Präsentation ausgelöst werden. Auch die Errichtung eines Festspielhauses durch einen Mäzen wird zu einem Komplementärprojekt, wenn die öffentliche Hand für den laufenden Betrieb und die Instandhaltung verantwortlich ist und dauerhaft Zuschüsse erforderlich sind.

Das wohl bekannteste Komplementärprojekt in Deutschland ist die Hamburger Elbphilharmonie, auch wenn die Grundidee hier zwar nicht aus dem mäzenatischen Bereich stammt. Vielmehr hatte ein privater Investor die Idee der Elbphilharmonie entwickelt, um ein eigenes Immobilienprojekt in der Nachbarschaft zu befördern bzw. eine Nutzung des Kaispeichers als Büroimmobilie zu verhindern (Faller und Wiegandt, 2015). Nachdem die ersten Mäzene ihre Un- 
terstützung für die überzeugende Idee der Elbphilharmonie zugesagt haben und Öffentlichkeit hergestellt wurde, ist die Stadt Hamburg unter Zugzwang geraten und nachträglich in den von privater Seite initiierten Planungsprozess eingestiegen. Unsere Interviews in Hamburg haben dabei zu der Einschätzung geführt, dass der Zeit- und Erwartungsdruck der Anfangsphase zu einer übereilten fehleranfälligen Planung beigetragen hat und sich daraus ein Teil der späteren Kostensteigerung, damit verbundene Kontroversen sowie Verzögerungen erklären lassen.

\subsection{Typ 4 - Projekte der öffentlichen Meinungs- und politischen Willensbildung}

Anders zu charakterisieren sind die vielfältigen Projekte, die primär auf die öffentliche Meinungs- und politische Willensbildung zielen. Die Finanzierung und Durchführung dieser Projekte ist meist unabhängig von der öffentlichen Hand und wirkt indirekt, indem die Wahrnehmung politischer Probleme beeinflusst oder konkrete Lösungen vorgeschlagen werden. Probate Mittel sind wissenschaftliche Studien, Kongresse und die Durchführung von Modellvorhaben zur Entwicklung und Erprobung von politisch relevanten Problemlösungen. Für stadtentwicklungspolitische Aufgabenstellungen sind in diesem Sinne z.B. die Schader-Stiftung (Darmstadt), die Wüstenrotstiftung (Ludwigsburg) oder die Montag Stiftung Urbane Räume (Bonn) aktiv. Aber auch die Bertelsmann Stiftung, der Generali Zukunftsfonds und viele weitere große Stiftungen berühren mit ihren Projekten und Themen auf vielfältige Weise stadt- und regionalentwicklungspolitische Fragestellungen. In der Regel können diese Projekte auch als Herausforderung an die Diskursfähigkeit der öffentlichen Hand interpretiert werden. Die vielfach geäußerte Kritik an der Bertelsmann Stiftung zeigt in diesem Kontext, dass insbesondere große Stiftungen mit ihren Möglichkeiten als relativ diskursbestimmend wahrgenommen werden.

\section{Schlussfolgerungen: Good Governance im Kontext mäzenatischen Engagements}

Die Kooperation von Mäzenen und Kommunen bringt besondere Herausforderungen mit sich. Als zentrales Ergebnis unserer Stiftungsbefragung ist festzuhalten, dass die Stiftungen, die im Verbund mit den Kommunen agieren, größere und spezifische Risiken für ihre Stiftungsarbeit sehen. Im Einzelnen verbindet sich dies mit folgenden Themen:

Die in einem kommunalpolitischen Umfeld agierenden Stiftungen antworten zu 36 Prozent, dass eine ,negative oder verzerrte öffentliche Wahrnehmung“" ein hohes Risiko für ihre Stiftungsarbeit darstellt (Vergleichswert 19\% für Stiftungen, die nicht in kommunalen Partnerschaften aktiv sind). In den Projektrecherchen wurden uns Beispiele genannt, bei denen mäzenatisches Engagement als Ergebnis sehr kontroverser öffentlicher Debatten mit sich artikulierendem Misstrauen und unterstellten unlauteren Absichten aus Sorge eines einseitigen Einflusses einzelner Akteure erlahmt ist. Da ein großer Teil der Stiftungen Wert darauf legt, öffentlich mit den Projekten identifiziert zu werden (55\% antworten in diesem Zusammenhang mit ,sehr wichtig“), entsteht in der öffentlichen Wahrnehmung und Debatte eine besondere Aufgabe für die Steuerung entsprechender Projekte. Kommunen, die mäzenatisches Engagement motivieren wollen, müssen sich in dieser Hinsicht als kompetent und zuverlässig erweisen.

Das Risiko einer ,mangelnden oder wegbrechenden Unterstützung von Dritten“ wird von 44 Prozent der in einem kommunalen Umfeld aktiven Stiftungen als hoch eingestuft. In der Praxis führt dies zum Wunsch der Mäzene, eigene Projekte möglichst hoch in der Verwaltungshierarchie anzusiedeln. Dies ist mit der Erwartung verbunden, dass hier getroffene Absprachen auch auf anderen Ebenen verbindlich sind bzw. Beachtung finden. Während dies in kleinen Städten oftmals möglich ist, werden insbesondere Großstädte ihre mit solchen Aufgaben betrauten Mitarbeiter mit entsprechenden Kompetenzen ausstatten müssen, um verlässliche Partnerschaften pflegen und philanthropisches Engagement motivieren zu können. Dabei bleibt die Frage zu diskutieren, wie solche Verbindlichkeiten dauerhaft mit einer demokratisch legitimierten Entscheidungsfindung etwa im Stadtrat vereinbar sind.

Zwischen den Mäzenen und den Städten und Gemeinden, in denen sie sich finanziell engagieren, gibt es unterschiedliche Formen des Zusammenspiels der beteiligten Akteure. Aus dem vielfältigen Alltag solcher Partnerschaften seien nur zwei Aspekte herausgegriffen:

Zum ersten haben wir Konstellationen beobachtet, in denen potentielle Mäzene heute eine reservierte Haltung gegenüber der örtlichen Stadtgesellschaft einnehmen. Sie haben erfahren, wie eine Stadtgesellschaft einen Mäzen in seinem Engagement nicht ausreichend gewürdigt und ihn durch eine schlechte Presse in eine unbefriedigende Rolle gebracht hat. Als Beispiel kann der Streit um die Namensgebung der Kunsthalle in Bielefeld dienen. Der Rat der Stadt hatte Ende der 1990er Jahre den zunächst über längere Zeit verwendeten Namenszusatz „Richard-Kaselowsky-Haus“ aus der Benennung der Halle gestrichen, weil der Stiefvater des Mäzens der Kunsthalle, Rudolf-August Oetker, eine aktive und damit kritische Rolle im Nationalsozialismus spielte. Dies hat über einen längeren Zeitraum zu öffentlich ausgetragenen Streitigkeiten in Bielefeld geführt, die potentielle Mäzene abgeschreckt haben und für ein Mäzenatentum in der Stadt nicht förderlich waren. In anderen Fällen haben sich Mäzene allerdings auch über vergleichbare öffentlich ausgetragene Streitigkeiten hinweggesetzt - so das schon erwähnte Ehepaar Greve in Hamburg im Fall der Flügelbauten der Universität. Angesichts der recht fest verankerten Tradition mäzenatischen Handelns scheinen solche Einzelfälle die positive Grundeinstellung der Bürgerschaft zum Mäzenatentum nicht zu erschüttern und das mäzenatische Engagement nicht spürbar zu beeinträchtigen. 
Zum zweiten gibt es Städte und Gemeinden, die sich inzwischen systematisch mit dem Phänomen des Mäzenatentums auseinandersetzen. Das bekannteste Beispiel ist die Stadt Hamburg, die als ,Stiftungshauptstadt“ in Deutschland gilt (Göring, 2007) und in der es schon seit Jahren verschiedene Aktivitäten gibt, das Stiftungswesen zu fördern. Dazu gehört etwa der lokale Stiftungstag, der als eine Art Informationsbörse bereits seit Ende der 1990er Jahre jährlich stattfindet (Inititativkreis Hamburger Stiftungen und Freie und Hansestadt Hamburg, 2003). Solche Aktivitäten gibt es auch in anderen Städten wie etwa in Gütersloh oder Bonn. In einigen Städten gibt es zudem eine systematische Unterstützung der Stadtverwaltungen, den Mäzenen Ansprechpartner für städtische Projekte zu vermitteln. So wissen Mäzene meist, in welchem thematischen Bereich sie sich engagieren wollen, wissen aber oft nicht, wie sie konkret helfen können. Hier ist eine Übersetzungsleistung der Städte hilfreich. In Nürnberg übernimmt dies etwa ein eigenes Amt in der kommunalen Verwaltung. In anderen Städten wurde betont, dass hierzu auch vertrauliche Gespräche zwischen der Stadtspitze und den Mäzenen geführt werden, um schon im Vorfeld eines Projektes mögliche Hindernisse bei der politischen Umsetzung auszuräumen. Mäzene sind also darauf bedacht, mit ihrem Handeln nicht in ein schlechtes öffentliches Licht zu geraten.

Zusammenfassend bleibt festzustellen, dass die Chancen des Mäzenatentums für die Stadtentwicklung in Deutschland angesichts des zunehmenden Reichtums einzelner Personen in einer neoliberalen Stadtgesellschaft steigen. Entscheidend für eine erfolgreiche Kooperation erscheint ein geregeltes Zusammenspiel von öffentlichen und privaten Akteuren mit einer Transparenz der Entscheidungsprozesse über den Einsatz der privaten Mittel. Dabei gibt es einige Hürden zu überwinden. Zum einen bedarf es eines geteilten Verständnisses darüber, dass die Kommunalpolitik heute nicht allein für das Gemeinwohl Verantwortung tragen kann und die Bedeutung des bürgerschaftlichen Engagements u.a. in Form des Mäzenatentums an Bedeutung für die Stadtentwicklung gewinnen wird. Zum anderen sind Mäzene aufgrund ihrer hybriden Stellung zwischen Markt und Zivilgesellschaft vielfach der Kritik ausgesetzt, nicht allein als gemeinwohlorientierte Akteure der Zivilgesellschaft aufzutreten. Aus einer eher kapitalismuskritischen Sicht treten sie gleichzeitig auch als Vertreter der neoliberalen Globalisierung auf, die als Privatpersonen mit ihrem erwirtschafteten Kapital nun einerseits dem Gemeinwohl etwas zurückgeben wollen, als private Akteure des Marktes aber durchaus Mitverursacher eben der sozialen Ungleichheit sind, die durch den Neoliberalismus verschärft wird. Die Erkenntnis, dass aufgrund der spezifischen Interessen der Mäzene Entscheidungsprozesse nicht immer transparent verlaufen und teilweise nicht den Zielsetzungen der Stadtpolitik entsprechen, fördert dabei die Diskussion um einseitige Einflussnahme und Demokratiedefizite. Hier zeigt sich, dass neue Formen der Aushandlung und Entscheidungsfindung zwischen den öffentlichen und privaten Akteu- ren notwendig sind, um die neuen Geschenke der Mäzene für das Gemeinwohl einzusetzen.

Danksagung. Die Autoren danken der Deutschen Forschungsgemeinschaft (DFG) für die Förderung des Projektes „Die geschenkte Stadt - Mäzenatentum in der deutschen Stadtentwicklung“. Des Weiteren danken die Autoren den Gutachtern für ihre wertvollen Hinweise, die zu einer Anreicherung und Verbesserung des Textes geführt haben

Edited by: B. Korf

Reviewed by: three anonymous referees

\section{Literatur}

Adloff, F.: Zivilgesellschaft: Theorie und politische Praxis, Campus, Frankfurt am Main, 2005.

Adloff, F.: Philanthropisches Handeln: Eine historische Soziologie des Stiftens in Deutschland und den USA, Campus, Frankfurt am Main, 2010.

Belina, B., Heeg, S., Pütz, R. und Vogelpohl, A.: Neuordnung des Städtischen im neoliberalen Zeitalter - Zur Einleitung, Geogr. Z., 101, 125-131, 2013.

Belina, B., Naumann, M. und Strüver, A. (Hrsg.): Handbuch Kritische Stadtgeographie, Westfälisches Dampfboot, Münster, 2014

Berndt, C.: Arbeiten und Produzieren, in: Schlüsselbegriffe der Kultur- und Sozialgeographie, Herausgeber: Lossau, J., Freytag, T. und Lippuner, R., Ulmer, Stuttgart, 227-242, 2014.

BMAS, Bundesministerium für Arbeit und Soziales (Hrsg.): Lebenslagen in Deutschland: Der vierte Armuts- und Reichtumsbericht der Bundesregierung, abrufbar unter: https://www.bmas. de/SharedDocs/Downloads/DE/PDF-Publikationen-DinA4/ a334-4-armuts-reichtumsbericht-2013.pdf?_blob= publicationFile (letzter Zugriff: 23. Oktober 2015), 2013.

Bundesverband Deutscher Stiftungen: Stadt trifft Stiftung: Gemeinsam gestalten vor Ort, StiftungsReport 2010, 11, Berlin, 2010.

Crouch, C.: Postdemokratie, Suhrkamp, Frankfurt am Main, 2008.

Crouch, C.: Das befremdliche Überleben des Neoliberalismus, Suhrkamp, Frankfurt am Main, 2011.

Deutscher Bundestag: Bürgerliches Engagement: auf dem Weg in eine zukunftsfähige Bürgergesellschaft, Drucksache, 14/8900, 2002.

Einig, K., Grabher, G., Ibert, O. und Strubelt, W.: Einführung Urban Governance, Informationen zur Raumentwicklung, H. 9/10, I-IX, 2005.

Faller, B.: Die städtische Philanthropie auf dem Weg ins Politische, polis - Magazin für Urban Development, 18, 38-43, 2011.

Faller, B. und Wiegandt, C.-C.: Die geschenkte Stadt: Mäzenatentum in der deutschen Stadtentwicklung, Forum Wohnen und Stadtentwicklung, 11, 327-334, 2010.

Faller, B. und Wiegandt, C.-C.: Die geschenkte Stadt - Mäzenatentum in der bundesdeutschen Stadtentwicklung, Berichte. Geographie und Landeskunde, 88, im Druck, 2015.

Fürst, D.: Die Stadt - neue Herausforderungen und Chancen für den Staat, dms - der moderne staat - Zeitschrift für Public Policy, Recht und Management, H1, 5, 25-42, 2012. 
Göring, M.: In Hamburg stiften gehen: Spaziergänge durch Deutschlands Stiftungshauptstadt, Ellert \& Richter, Hamburg, 2007.

Häußermann, H., Läpple, D., und Siebel, W.: Stadtpolitik, Suhrkamp, Frankfurt am Main, 2008.

Heinz, W.: (Ohn-)mächtige Städte in Zeiten der neoliberalen Globalisierung, Westfälisches Dampfboot, Münster, 2015.

Hohn, U., Lötscher, L. und Wiegandt, C.-C.: Governance - ein Erklärungsansatz für Stadtentwicklungsprozesse, in: Berichte zur deutschen Landeskunde 80, H. 1, S. 5-15, 2006.

Initiativkreis Hamburger Stiftungen und Freie und Hansestadt Hamburg: Einleitung, in: Bürger und Gesellschaft: Stiftungen in Hamburg, Initiativkreis Hamburger Stiftungen, Herausgeber: Meyer, S., Hamburg, 10-11, 2003.

Klein, S.: Der Sinn des Gebens, Fischer, Frankfurt am Main, 2010.

Krimphove, P.: „Es wird hier einfach erwartet“ - Philanthropie in den USA und in Deutschland, in: Vermögenskultur: Verantwortung im 21. Jahrhundert, Herausgeber: Druyen, T., VS Verlag für Sozialwissenschaften, Wiesbaden, 131-152, 2011.

Lauterbach, W., Hartmann, M. und Ströing, M. (Hrsg.): Reichtum, Philanthropie und Zivilgesellschaft, Springer VS, Wiesbaden, 2014.

Mattissek, A. und Prossek, A.: Regieren und Planen, in: Schlüsselbegriffe der Kultur- und Sozialgeographie, Herausgeber: Lossau, J., Freytag, T. und Lippuner, R., Ulmer, Stuttgart, 198-211, 2014.

Müller-Jentsch, D.: Schweizer Stiftungswesen im Aufbruch, aufrufbar unter: http://www.avenir-suisse.ch/wp-content/uploads/ 2014/10/schweizer_stiftungswesen_hp3.pdf (letzter Zugriff: 23. Oktober 2015), 2014.

Ostrower, F.: Why the Wealthy Give: The Culture of Elite Philanthropy, Princeton, 1995.
Priller, E. und Sommerfeld, J.: Wer spendet in Deutschland? Eine sozialstrukturelle Analyse, WZB Discussion Paper, 202, Berlin, abrufbar unter: http://skylla.wzb.eu/pdf/2005/i05-202.pdf (letzter Zugriff: 23. Oktober 2015), 2005.

Quaestio Forschung und Beratung (Hrsg.): Gesellschaftliches Engagement von Unternehmen und Stiftungen in der sozialen Quartiersentwicklung, Diskussions- und Positionspapier des Expertenbeirates im ExWoSt-Forschungsfeld „Unternehmen und Stiftungen für die soziale Quartiersentwicklung“, Bonn, abrufbar unter: http://www.quaestio-fb.de/ files/gesellschaftliche_engagement_von_unternehmen_und_ stiftungen_in_der_sozialen_quartiersentwicklung.pdf (letzter Zugriff: 23. Oktober 2015), 2015.

Rickens, C.: Ganz oben: Wie Deutschlands Millionäre wirklich leben, Kiepenheuer \& Witsch, Köln, 2011.

Selle, K.: Planen, Steuern, Entwickeln. Über den Beitrag öffentlicher Akteure zur Entwicklung von Stadt und Land, Edition StadtEntwicklung, Verlag Dorothea Rohn, Dortmund, 2005.

Selle, K.: Über Bürgerbeteiligung hinaus: Stadtentwicklung als Gemeinschaftsaufgabe? Analysen und Konzepte, Edition StadtEntwicklung, Verlag Dorothea Rohn, Detmold, 2014.

Strachwitz, R.: Der zivilgesellschaftliche Mehrwert. Eine Einführung, Opusculum, 39, 5-20, abrufbar unter: http://www.institut. maecenata.eu/resources/091214-op39.pdf (letzter Zugriff: 23. Oktober 2015), 2009.

Timmer, K.: Stiften in Deutschland: Die Ergebnisse der StifterStudie, 2. Ausgabe, Verlag Bertelsmann-Stiftung, Gütersloh, 2006.

Wiegandt, C.-C.: Macht - Stadt (statt) - Planung, Berichte zur deutschen Landeskunde 75, H. 2/3, S. 320-332, 2001.

Wiegandt, C.-C.: Stadtentwicklung in Deutschland: Disparitäten und Polarisierung als aktuelle Themen der Stadtentwicklung, Praxis Geographie, 45, H. 3, 4-11, 2015. 